\title{
INFLUENCIA DO PERICARPO NO TESTE DE CONDUTIVIDADE ELÉTRICA EM SEMENTES DE CRAMBE (Crambe abyssinica HOCHST) SUBMETIDAS A MÉTODOS DE SECAGEM
}

\author{
Magnun Antonio Penariol da Silva ${ }^{1}$, Marco Antonio Martin Biaggioni ${ }^{2}$, Felipe Carlos Spneski \\ Sperotto $^{3}$, Arthur Celidonio Heiland Puteri ${ }^{4} \&$ Reni Saath $^{5}$
}

RESUMO: O presente trabalho teve como objetivo avaliar o efeito de cinco sistemas de secagem sobre a qualidade de sementes de crambe e a influência do pericarpo no teste de condutividade elétrica. O campo de sementes foi conduzido na Faculdade de Ciências Agronômicas, UNESP - Univ Estadual Paulista. O delineamento experimental foi em blocos casualizados com quatro repetições. Foram empregados os seguintes métodos de secagem: secagem no campo, no terreiro, à sombra, com ar aquecido e com ar não aquecido. As sementes utilizadas foram da cultivar FMS Brilhante. Utilizou-se para avaliar a qualidade das sementes a análise da acidez graxa e o teste de condutividade elétrica, com e sem pericarpo. Após obtenção dos dados procedeu-se a análise de variância, e as médias foram comparadas pelo teste de Tukey $(\mathrm{p} \leq 0,05)$. Foi realizado também o teste de correlação linear de Pearson entre os testes de condutividade elétrica e acidez graxa visando avaliar a influência do pericarpo. Concluiu-se que o sistema de secagem à sombra causa menores danos às sementes e que a presença do pericarpo nas sementes de crambe diminui a sensibilidade do teste de condutividade elétrica.

PALAVRAS-CHAVE: Biodiesel; acidez graxa; semente oleaginosa.

\section{INFLUENCE OF THE PERICARP IN ELECTRICAL CONDUCTIVITY OF CRAMBE (Crambe abyssinica HOCHST) SEEDS SUBMITTED TO DIFFERENT DRYING SYSTEMS}

\begin{abstract}
In this study, we aimed to evaluate the effect of five drying systems on the quality of crambe seed and the influence of the pericarp in its electrical conductivity. The experimental design adopted was randomized blocks with four replications. The following drying methods were applied: drying in the field, on the patio, under shade, with heated air, and non-heated air. To evaluate the electrical conductivity and the fat acidity, the seeds, from the cultivar FMS Brilhante, were analyzed with and without pericarp. . An analysis of variance was performed and the means were compared by Tukey test $(\mathrm{p} \leq 0,05)$. We also performed the Pearson linear correlation between electrical conductivity and fat acidity in order to evaluate the pericarp influence. The 4 drying system using shades cause less damage to the seeds; and the presence of pericarp decreased the sensitivity of the electrical conductivity.
\end{abstract}

KEYWORDS: Biodiesel; fat acidity, oilseed.

\footnotetext{
1 UNESP/FCA. Mestre em Agronomia (Energia na Agricultura). E-mail: penariol@gmail.com

2 UNESP/FCA. Professor do Departamento de Engenharia Rural. E-mail: biaggioni@fca.unesp.br

3 Mestre em Agronomia (Energia na Agricultura). E-mail: felipesperotto@hotmail.com
}

\begin{tabular}{l}
\hline Graduando em $\quad$ Agronomia. \\
arthur.celidonio@hotmail.com \\
${ }^{5}$ Doutora em Agronomia (Energia na Agricultura). E-mail: \\
reniagricola@yahoo.com.br
\end{tabular}




\section{INTRODUÇÃO}

O crambe (Crambe abyssinica Hochst. ex Fries) é uma planta de ciclo anual, pertencente à família Brassicaceae cujas sementes contêm cerca de $35-60 \%$ de óleo. É nativa do Mediterrâneo e cultivada em algumas regiões tropicais e subtropicais pelo interesse industrial no óleo extraído destas sementes, e mais recentemente, para produção de óleo para biodiesel (CARNEIRO et al., 2008).

O crambe se mostra como uma interessante fonte de óleo vegetal, cerca de $44,1 \%$ na massa seca, sendo assim uma cultura promissora para a produção de biodiesel (BERALDO et al., 2010; SOUZA et al., 2009). A produção de crambe tem como vantagens o cultivo totalmente mecanizado, utilizando os mesmos equipamentos existentes para outras culturas, e a possibilidade de cultivo no inverno. Por ser uma cultura pouco conhecida comercialmente, praticamente não se dispõe, ainda, de informações técnicas que viabilize seu cultivo intensivo (OLIVA et al., 2012; PITOL et al., 2010). É tolerante à geada e, após seu estabelecimento, é altamente resistente à seca. Por apresentar boa produtividade na estação seca, tem-se mostrado uma boa alternativa para a safrinha, constituindo-se numa opção para a rotação de culturas (MEAKIN, 2001).

Em estudos realizados, observou-se um potencial de produtividade do crambe em torno de 1000 a $1500 \mathrm{~kg} \mathrm{ha}$

(FUNDAÇÃO MS, 2007). Brandão et al. (2013) encontraram produtividade média de $1428,98 \mathrm{~kg} \mathrm{ha}^{-1}$. Jasper (2010), em experimento de crambe produzido em plantio direto em Botucatu-SP, encontrou produtividade média de $1507,05 \mathrm{Kg} \mathrm{ha}^{-1}$ o que demonstra boa aptidão da cultura à região.

Um fator determinante para o sucesso no processo póscolheita de produtos agrícolas é o sistema de secagem a ser empregado nas sementes. A escolha do método correto implicará em menores perdas na produção e melhor qualidade do produto. Quando realizado de maneira adequada, o manejo pós-colheita resulta em maiores ganhos para a produção, pois evita a contaminação dos produtos com pragas e doenças e ainda evita a perda da sua qualidade.

Um método utilizado para avaliar a qualidade das sementes, que tem resposta rápida e de baixo custo é a análise da acidez graxa, que quantifica a deterioração de grãos ainda em seu estágio inicial.

A principal vantagem do teste de acidez graxa, como método para avaliar a deterioração em grãos/sementes armazenados é a sua sensibilidade. Entre as primeiras reações que acontecem sob condições adversas no manejo pós-colheita, está a formação de ácidos graxos livres, que ocorre por causa da hidrólise das gorduras. A análise dessa reação permite identificar o processo deteriorativo das sementes ainda em seu estágio inicial e fazer também sua quantificação (SOARES, 2005).
O teste de condutividade elétrica baseia-se no princípio de que com o processo de deterioração ocorre a lixiviação dos constituintes celulares das sementes embebidas em água devido à perda da integridade dos sistemas celulares. Desse modo, baixa condutividade elétrica significa alta qualidade da semente e alta condutividade, ou seja, maior saída de lixiviados da semente sugere o menor vigor desta (VIEIRA \& KRZYZANOWSKI, 1999).

Albuquerque et al. (2001) avaliaram a metodologia do teste de condutividade elétrica nas sementes de quatro genótipos de girassol (IAC-Iarama, V-2000, M-742 e C11), utilizando cinco períodos de embebição $(16,20,24$, 28 e 32 horas), nas temperaturas de 25 e $30{ }^{\circ} \mathrm{C}$, em quatro repetições de 50 sementes. Avaliaram-se, também, a condutividade elétrica da solução obtida dos aquênios (sementes com pericarpo), sementes sem pericarpo e pericarpos isolados (cascas) de girassol. Concluíram que sementes sem o pericarpo apresentam melhor qualidade em relação aos resultados de condutividade elétrica.

O pericarpo é uma estrutura lignificada formada por substâncias capazes de produzir um aumento da condutividade e a sua presença prejudica a avaliação do estado deteriorativo das membranas de girassol, pelo teste de condutividade elétrica, induzindo a erros de interpretação (LONGO et al. 1999)

Segundo estudos realizados avaliando o efeito imediato do método de secagem na qualidade de sementes de crambe, o teste de condutividade elétrica não detectou diferença na qualidade das sementes entre os diferentes tipos de secagem. Deve ser considerada no teste de condutividade elétrica em sementes de crambe a presença do pericarpo, pois estima-se que, a presença do mesmo dificulta a completa submersão da amostra durante o período de repouso, impedindo o contato de toda a superfície da semente pela solução de embebição (OLIVA et al. 2012).

Silva et al. (2013), avaliando o efeito imediato da secagem na cultura do crambe, encontraram valores de acidez graxa variando entre 6,7 (mL KOH 0,1 N 100g MS) para secagem com aquecimento de ar, e 9,1 (mL $\left.\mathrm{KOH} \quad 0,1 \quad \mathrm{~N} \quad 100 \mathrm{~g}^{-1} \mathrm{MS}\right)$ para secagem realizada naturalmente na planta. A cultura do crambe apresenta custos operacionais elevados em relação ao seu armazenamento e transporte devido a baixa densidade de estocagem do grão, estimada em $328 \mathrm{~kg} / \mathrm{m}^{3}$ decorrente da espessura da casca de $(0,23 \mathrm{~mm})$, que caracteriza $21,2 \%$ do peso da semente. Estes custos poderiam ser reduzidos com o descascamento da semente, bem como incremento no teor protéico do coproduto (casca). Porém, há falta de informações adequadas sobre a qualidade do óleo extraído de sementes sem casca (REUBER et al., 2001).

Segundo Plein et al (2010), avaliando as características de qualidade do óleo extraído de grãos de crambe sem 
casca comparados ao com casca armazenados durante 42 dias, concluíram que a estabilidade oxidativa não foi influenciada pela ausência ou presença da casca, no período avaliado.

O objetivo do presente trabalho foi avaliar o efeito do pericarpo no teste de condutividade elétrica em sementes de crambe.

\section{MATERIAL E MÉTODOS}

O experimento foi instalado na Fazenda Experimental Lageado pertencente à Faculdade de Ciências Agronômicas - Universidade Estadual Paulista "Júlio de Mesquita Filho"/ Câmpus de Botucatu.

Os sistemas de secagem empregados consistiram em secagem no campo, no terreiro, secagem artificial com ar aquecido, secagem artificial com ar não aquecido e, como testemunha, a secagem à sombra. Foram utilizadas sementes de crambe da cultivar FMS Brilhante, armazenadas por 18 meses em embalagens de papel, em ambiente natural. $\mathrm{O}$ delineamento experimental empregado foi em blocos casualizados, com quatro repetições.

No tratamento de secagem no campo, as plantas permaneceram na lavoura até atingirem teor de água próximo a $10 \%$ b.u., monitorado pelo método da estufa a $105 \pm 3{ }^{\circ} \mathrm{C}$ (BRASIL, 2009). Na secagem em terreiro, as sementes foram distribuídas em terreiro de tijolo maciço numa camada de $5 \mathrm{~cm}$ de espessura, revolvidas periodicamente e amontoadas e cobertas com lona no final do dia. A secagem com ar aquecido foi realizada em um secador com quatro colunas de PVC de $0,8 \mathrm{~m}$ de altura e $0,15 \mathrm{~m}$ de diâmetro, a temperatura média no duto de entrada de ar foi de $45,5^{\circ} \mathrm{C}$, temperatura média na massa de sementes de $39,7^{\circ} \mathrm{C}$ e fluxo de ar de $217,3 \mathrm{~m}^{3}$ $\min ^{-1}$ ton $^{-1}$. A altura da massa de grãos em cada coluna foi de 0,60 metros, e o ar de secagem foi insuflado no sistema por meio de um ventilador acionado por um motor elétrico. A secagem com ar não aquecido foi realizada em um secador de leito fixo com quatro colunas de PVC de 1,5 m de altura e $0,15 \mathrm{~m}$ de diâmetro, com uma tela metálica no fundo, a temperatura média na massa de sementes e no duto de entrada de ar foi de $21 \mathrm{e}$ $24{ }^{\circ} \mathrm{C}$, respectivamente, e o fluxo de ar foi de 25,7 $\mathrm{m}^{3} \cdot \mathrm{min}^{-1}$ ton $^{-1}$. A altura da massa de grãos em cada coluna foi de 1,25 metros. Para secagem à sombra, as sementes foram espalhadas com espessura de apenas uma semente sobre lona plástica e mantidas sob telhado com ventilação natural.

A análise da acidez graxa (AG) foi realizada conforme a recomendação de AACC (1995). A acidez graxa foi determinada pela seguinte equação: $A G=$ volume de $\mathrm{KOH} /$ peso da amostra seca.

Para o teste de condutividade elétrica, foram separadas as sementes com e sem pericarpo (casca) e a avaliação do efeito se deu com o uso 100 sementes, embebidas por 16 horas, em $50 \mathrm{ml}$ de água deionizada, de acordo com a metodologia utilizada para canola (WAGNER \& DUCOURNAU, 2007).

Após obtenção dos dados procedeu-se a análise de variância e as médias foram comparadas pelo teste de Tukey $(p \leq 0,05)$, utilizando o programa Sisvar da Universidade Federal de Lavras (FERREIRA, 2000).

Foi realizado também, o teste de correlação de Pearson utilizando o programa Sanest da Universidade Federal de Pelotas (ZONTA et al., 1986).

\section{RESULTADOS E DISCUSSÃO}

De acordo com os resultados obtidos, conforme apresentados na Tabela 1, o menor índice de ácidos graxos livres foi encontrado na secagem à sombra (testemunha). Em relação ao teste de condutividade elétrica, não houve diferença significativa entre os sistemas de secagem quando as sementes estavam envolvidas pelo pericarpo.

\begin{tabular}{|c|c|c|c|}
\hline abela 1 & \multicolumn{3}{|c|}{$\begin{array}{l}\text { Valores médios de acidez graxa } \\
\left.\text { (mL KOH 0,1 N } 100 g^{-1} M S\right) \text { e } \\
\text { condutividade elétrica }(C E-\mu S \mathrm{~cm}-1 \mathrm{~g} \text { - } \\
\text { 1) com ausência (CP) e presença (SP) } \\
\text { do pericarpo em função do sistema de } \\
\text { secagem. }\end{array}$} \\
\hline $\begin{array}{l}\text { Sistemas de } \\
\text { secagem }\end{array}$ & $\begin{array}{c}\text { Acidez } \\
\text { Graxa }\end{array}$ & $\mathbf{C E}-\mathbf{C P}$ & CE - SP \\
\hline Ar aquecido & $36,7100 \mathrm{~b}$ & $170,5375 \mathrm{a}$ & $180,1200 \mathrm{~b}$ \\
\hline $\begin{array}{l}\text { Ar não } \\
\text { aquecido }\end{array}$ & $36,0275 \mathrm{~b}$ & $177,3500 \mathrm{a}$ & $169,1975 \mathrm{ab}$ \\
\hline Terreiro & $29,9575 \mathrm{~b}$ & $175,0925 \mathrm{a}$ & $168,6750 \mathrm{ab}$ \\
\hline Campo & $31,7250 \mathrm{~b}$ & 149,5375 a & $138,2250 \mathrm{ab}$ \\
\hline Sombra & $17,575 \mathrm{a}$ & $172,1950 \mathrm{a}$ & $130,1000 \mathrm{a}$ \\
\hline $\mathrm{F}$ & $44,88^{*}$ & $1,98^{\mathrm{NS}}$ & $113,89^{*}$ \\
\hline C.V. (\%) & 7,51 & 2,26 & 2,61 \\
\hline
\end{tabular}

NS: Não significativo ( $p \leq 0,05)$; *Significativo $(p \leq 0,05)$ C.V.: Coeficiente de variação

Médias seguidas pela mesma letra não diferem entre si pelo teste de Tukey $(p \leq 0,05)$

Para as sementes com ausência de pericarpo o menor índice de lixiviados também foi encontrado na secagem à sombra, diferindo significativamente da secagem em alta temperatura, mas não da secagem no campo, no terreiro e em baixa temperatura.

Para o teste de condutividade elétrica, as sementes envolvidas pelo pericarpo mostraram menor sensibilidade ao teste, igualando estatisticamente, todos os tratamentos aplicados. Quando o teste foi conduzido sem o pericarpo, houve maior sensibilidade, permitindo 
diferenciar, estatisticamente, os tratamentos com secagem em alta temperatura e à sombra.

Essa maior sensibilidade nas sementes sem o pericarpo, corroboram os resultados obtidos por Brandão Júnior et al. (1997), que encontraram maior eficácia no teste de condutividade elétrica em sementes de girassol sem o pericarpo.

A Tabela 2 apresenta os resultados do teste de correlação visando avaliar a influência do pericarpo nas análises de condutividade elétrica das sementes de crambe.

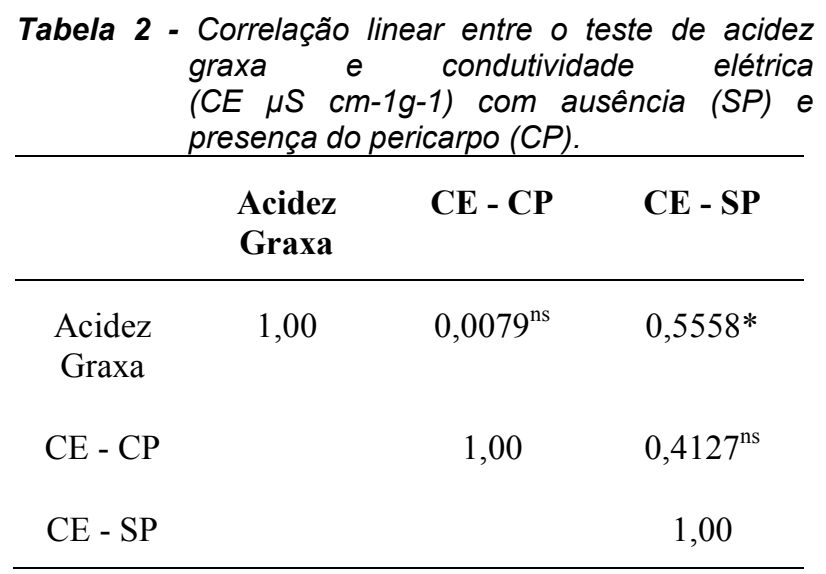

${ }^{\mathrm{ns}}$ Não significativo

*Significativo a 5\% de significância

Pelos dados, observa-se que houve uma correlação positiva e significativa apenas entre as variáveis acidez graxa e condutividade elétrica sem o pericarpo, enquanto o teste de condutividade elétrica conduzido em crambe com casca não se correlacionou significativamente nem com a acidez graxa nem com a condutividade elétrica em sementes descascadas.

Como a análise da acidez graxa tem como uma das suas principais características a alta sensibilidade, a correlação positiva com o teste de condutividade elétrica sem o pericarpo, demonstra que a retirada do pericarpo antes da embebição das amostras constitui-se num procedimento recomendável, possibilitando obter resultados mais confiáveis nas avaliações da qualidade de sementes de crambe.

Os dois testes de qualidade empregados, acidez graxa e condutividade elétrica em sementes sem pericarpo (SP), indicam o efeito do armazenamento nos tratamentos de secagem, em relação ao tratamento testemunha (secagem à sombra).

Os maiores valores de acidez graxa e condutividade elétrica (SP), sugerindo uma maior deterioração da semente armazenada, foram encontrados no tratamento que secou com ar aquecido. Embora diferindo estatisticamente da testemunha, este tratamento não diferiu estatisticamente dos demais. Possivelmente, a temperatura na massa de sementes, em torno de $40{ }^{\circ} \mathrm{C}$, não se revelou tão agressiva a ponto e comprometer a qualidade fisiológica do crambe. Estes resultados concordam com os obtidos por Donadon et al. (2012) que, estudando o efeito da secagem de crambe em estufa de ventilação forçada. Nas temperaturas de 35, 45, 60 e $75{ }^{\circ} \mathrm{C}$, concluíram não terem ocorrido danificações no pericarpo.

Por outro lado, o bom desempenho dos tratamentos que empregaram ar não aquecido, não diferindo significativamente da testemunha, pode ser explicado pela época favorável à secagem com ar natural na região de Botucatu-SP, a qual coincidiu com um período sem ocorrência de chuvas e com umidade relativa média de $65 \%$. Entre os tratamentos que utilizaram ar não aquecido, o tempo de secagem atingiu 4, 5 e 6 dias, respectivamente, para os sistemas de secagem em terreiro, com ar natural e na planta.

\section{CONCLUSÕES}

Conclui-se que todos os tratamentos de secagem apresentaram um efeito latente sobre a qualidade da semente, em relação à secagem à sombra (testemunha), e que a retirada do pericarpo, antes do teste de condutividade elétrica, aumenta a sensibilidade e confiabilidade da análise.

\section{REFERÊNCIAS}

ALBUQUERQUE, M. C. F. E.; MORO, F. V.; FAGIOLI, M.; RIBEIRO, M. C. Condutividade elétrica e lixiviação do potássio para avaliar sementes de girassol. Revista Brasileira de Sementes, Londrina, v. 23, n. 1, p. 1-8, 2001.

\section{AMERICAN ASSOCIATION OF CEREAL CHEMISTS. Approved methods of the AACC. 8. ed.} Saint Paul: AACC, 1995.

BERALDO, J. M. G. Determinação da época de semeadura do Crambe (Crambe abyssinica Hochst) na região nordeste do Estado de São Paulo-SP. In: CONGRESSO DA REDE BRASILEIRA DE BIODIESEL, 4.; CONGRESSO BRASILEIRO DE PLANTAS OLEAGINOSAS, ÓLEOS, GORDURAS E BIODIESEL, 7, Varginha. Anais... Belo Horizonte: 2010 .

BRANDÃO, F. J. B.; SILVA, A. R. B.; SILVA, M. A. P.; SPEROTTO, F. C. S. Desempenho operacional e produtividade agrícola do crambe nos preparos convencional e reduzido de solo. In: ENCICLOPÉDIA BIOSFERA. Goiânia: Centro Científico Conhecer, 2013. v. 9, n. 17, p. 2013.

BRANDÃO JUNIOR, D. S.; RIBEIRO, D. C. A.; BERNADINO FILHO, J. R.; VIEIRA, M. G. C. C. Adequação do teste de condutividade elétrica para determinar a qualidade fisiológica de sementes. 
Informativo ABRATES, Curitiba, v. 7, n. 1/2, p. 184, 1997.

BRASIL. Ministério da Agricultura, Pecuária e Abastecimento. Regras para análise de sementes. 2009. 399 p.

CARNEIRO, S. M. T. P .G.; ROMANO, E.; MARIANOWSKI, T.; OLIVEIRA, J. P.; GARBIM, T. H. DOS S.; ARAÚJO, P. M. Ocorrência de Alternaria brassicicola em crambe (Crambe abyssinica) no estado do Paraná. Comunicações. Summa Phytopathologica, Botucatu, v. 35, n. 2, p. 154, 2009. Disponível em: $<$ http://dx.doi.org/10.1590/S0100-

54052009000200016>. Acesso em: 4 ago. 2012. doi: $10.1590 / \mathrm{S} 0100-54052009000200016$

DONADON, J. R.; RESENDE, O.; TEIXEIRA, S. P.; SANTOS, J. M.; MORO, F. V. Danificação pela secagem em frutos e sementes de crambe. In: CONGRESO LATINOAMERICANO Y DEL CARIBE DE INGENIERÍA AGRÍCOLA, 10; CONGRESSO BRASILEIRO DE ENGENHARIA AGRÍCOLA, 41., 2012, Londrina. In: Anais...Londrina: 2012.

FERREIRA, F. A. Sistema SISVAR para análises estatísticas. Lavras: Universidade Federal de Lavras 2000. Disponível em:

$<$ http://www.dex.ufla.br/ danielff/softwares.htm>. Acesso em: 7 abr. 2011.

FUNDAÇÃO MATO GROSSO DO SUL. Crambe FMS Brilhante. Maracajú, 2007. Disponível em:

$<$ http://www.fundacaoms.org.br/page.php?34>. Acesso em: 11 de outubro de 2011.

JASPER, S. P. Análise energética da cultura do crambe (Crambe abyssinica Hochst) produzida em plantio direto. Engenharia Agrícola, Jaboticabal, v. 30, n. 3, p. 395403, maio/jun. 2010.

LONGO, O.; PÉREZ, A. H.; MURCIA, M. Efecto de la presencia de pericarpio sobre los valores de conductividad en semillas de girasol (Helianthus annuus L.) com diferentes niveles de deterioro. Informativo ABRATES, Curitiba, v. 9, n. 1/2, p. 149, 1999.

MEAKIN, S. Crambe abyssinica: a comprehensive programme. Rudston: Springdale Crop Synergues Ltda, 2001.

OLIVA, A. C. E.; BIAGGIONI, M. A. M.; CAVARIANI, C. Efeito imediato do método de secagem na qualidade de sementes de crambe. Energia na Agricultura, Botucatu, v. 27, n. 3, p.16-30, jul./set. 2012.
PITOL, C.; BROCH, D. L.; ROSCOE, R. Tecnologia e produção: Crambe. Maracaju: Fundação MS, 2010. 60 p.

PLEIN, G. S.; FAVARO, S. P.; SOUZA, A. D. V.; SOUZA, C. F. T.; CICONINI, G.; SANTOS, G. P.; MIYAHIRA, M. A. M.; ROSCOE, R. Caracterização da fração lipídica em sementes de crambe armazenadas com e sem casca. In: CONGRESSO BRASILEIRO DE MAMONA, 4.; SIMPÓSIO INTERNACIONAL DE OLEAGINOSAS ENERGÉTICAS, 1., 2010, João Pessoa. Inclusão Social e Energia: Anais. Campina Grande: Embrapa Algodão, 2010. p. 1812-1816.

REUBER, M. A.; JOHNSON, L.A.; WATKINS, L. R. Dehulling crambe seed for improved oil extraction and meal quality. Journal of the American Oil Chemist's Society, v. 78, n. 6, p. 661-664, 2001.

SOUZA, A. D. V.; FÁVARO, S. P.; ÍTAVO, L. C. V.; ROSCOE, R. Caracterização química de Sementes e tortas de pinhão-manso, nabo-Forrageiro e crambe. Pesquisa agropecuária brasileira, Brasília, v. 44, n. 10, p. 1328-1335, 2009. Disponível em: $<\mathrm{http}$ :/dx.doi.org/10.1590/S0100204X2009001000017>. Acesso em: 3 ago. 2012.

SILVA, M. A. P.; BIAGGIONI, M. A. M.; SPEROTTO, F. C. S.; BEZERRA, P. H. S.; BRANDÃO, F. J. B. Qualidade do oleo bruto de grãos de crambe (Crambe abyssinica Hochst) sob diferentes métodos de secagem. Energia naAgricultura, Botucatu, v. 28, n. 3, p. 193199, jul./set. 2013.

SOARES, T. A.; BIAGGIONI, M. A. M.; FRANÇA NETO, J. B. Análise da acidez graxa como índice de qualidade em grãos de soja. Energia na Agricultura, Botucatu, v. 20, n. 1, p. 91-102, 2005.

VIEIRA, R. D.; KRZYZANOWSKI, F. C. Teste de condutividade elétrica. In: KRZYZANOWSKI, F. C.; VIEIRA, R. D.; FRANÇA NETO, J. B. (Ed.). Vigor de sementes: conceitos e testes. Londrina: ABRATES, 1999. cap. 4, p. 1-26.

WAGNER, M. H.; DUCOURNAU, S. Conductivity testing for oilseed rape seeds. ISTA News Bulletin, n. 133, Apr., 2007. Disponível em: $<$ http://www.seedtest.org/upload/cms/user/STI133April2 007.pdf $>$. Acesso em: 13 nov. 2011.

ZONTA, E. P., SILVEIRA, P.; MACHADO, A. A. Sistema de análise estatística para microcomputadores - SANEST. Pelotas: Instituto de Física e Matemática, Universidade Federal de Pelotas, 1986 